\title{
Sudden motility reversal indicates sensing of magnetic field gradients in Magnetospirillum magneticum AMB-1 strain
}

\author{
Lina M González ${ }^{1}$, Warren C Ruder ${ }^{2}$, Aaron P Mitchell ${ }^{3}$, William C Messner ${ }^{1,4}$ \\ and Philip R LeDuc ${ }^{1}$ \\ ${ }^{1}$ Department of Mechanical Engineering, Carnegie Mellon University, Pittsburgh, PA, USA; ${ }^{2}$ Department of \\ Biological Systems Engineering, Virginia Tech, Blacksburg, VA, USA; ${ }^{3}$ Department of Biological Science, \\ Carnegie Mellon University, Pittsburgh, PA, USA and ${ }^{4}$ Department of Mechanical Engineering, Tufts \\ University, Medford, MA, USA
}

\begin{abstract}
Many motile unicellular organisms have evolved specialized behaviors for detecting and responding to environmental cues such as chemical gradients (chemotaxis) and oxygen gradients (aerotaxis). Magnetotaxis is found in magnetotactic bacteria and it is defined as the passive alignment of these cells to the geomagnetic field along with active swimming. Herein we show that Magnetospirillum magneticum (AMB-1) show a unique set of responses that indicates they sense and respond not only to the direction of magnetic fields by aligning and swimming, but also to changes in the magnetic field or magnetic field gradients. We present data showing that AMB-1 cells exhibit sudden motility reversals when we impose them to local magnetic field gradients. Our system employs permalloy $\left(\mathrm{Ni}_{80} \mathrm{Fe}_{20}\right)$ islands to curve and diverge the magnetic field lines emanating from our custom-designed Helmholtz coils in the vicinity of the islands (creating a drop in the field across the islands). The three distinct movements we have observed as they approach the permalloy islands are: unidirectional, single reverse and double reverse. Our findings indicate that these reverse movements occur in response to magnetic field gradients. In addition, using a permanent magnet we found further evidence that supports this claim. Motile AMB-1 cells swim away from the north and south poles of a permanent magnet when the magnet is positioned less than $\sim 30 \mathrm{~mm}$ from the droplet of cells. All together, these results indicate previously unknown response capabilities arising from the magnetic sensing systems of AMB-1 cells. These responses could enable them to cope with magnetic disturbances that could in turn potentially inhibit their efficient search for nutrients.
\end{abstract}

The ISME Journal (2015) 9, 1399-1409; doi:10.1038/ismej.2014.224; published online 5 December 2014

\section{Introduction}

Diverse organisms can sense and respond to the Earth's magnetic field. This ability is dependent upon the intracellular biomineralization of magnetic particles and the formation of organic compartments or vesicles; this magnetic field sensing behavior can be found in a diversity of organisms (Lowenstam, 1981). For example, Gould et al. (1978) discovered that honeybees contain magnetite localized in their abdomen, and Walcott and colleagues (Walcott, 1977; Walcott et al., 1979) discovered magnetite in the head of homing

Correspondence: P LeDuc, Department of Mechanical Engineering, Carnegie Mellon University, 5000 Forbes Avenue, Pittsburgh, PA 15213, USA or W Messner, Department of Mechanical Engineering, Tufts University, 204 Anderson Hall, Medford, MA 02155, USA.

E-mail: pr@@andrew.cmu.edu or william.messner@tufts.edu

Received 6 May 2014; revised 14 September 2014; accepted 23

October 2014; published online 5 December 2014 pigeons. Among other eukaryotic organisms in which magnetite has been experimentally found are fish (Mann et al., 1988; Diebel et al., 2000) and termites (Maher 1998). All of these organisms presumably use this mineral as a 'compass' to sense magnetic fields when navigating and homing.

Certain unicellular microorganisms such as magnetotactic bacteria (MTB) have the ability to synthesize chains of mineral crystals (Ruder et al., 2012), each of which is enveloped by individual lipid bilayers in organelles known as magnetosomes (Frankel et al., 1979). These magnetosome inclusions render these creatures a model organism to study compartmentalization in prokaryotes (Komeili, 2012), and the formation mechanisms of these structurally organized magnetosomes under genetic control have been elucidated (Komeili et al., 2004, 2006; Scheffel et al., 2006). According to previous studies the proposed mechanisms of the assembly of magnetosomes are: (1) the magnetosome 
compartment invaginates from the inner cell membrane (Komeili et al., 2004); (2) individual magnetosomes are recruited to the actin-like protein, MamK (Komeili et al., 2006; Katzmann et al., 2010) (providing a structural scaffold), where an acidic protein, MamJ (Scheffel et al., 2006), (running along the cell, from pole to pole), attaches them to this cytoskeleton filaments in a beads-on-a-string manner; (3) ferric ions are chelated by siderophores and transported into these cells; and (4) magnetite crystallization ensues within the nucleated vesicles. There is a plethora of information about magnetosomes and the proteins involved in their formation processes. However, there is no study showing evidence of the ability of magnetotactic bacteria to cope with magnetic disturbances or fluctuations (that is, gradients) in their surrounding environments that can misguide them, pull them and inhibit their efficient search for nutrients. Investigation of the swimming behavior in the presence of magnetic field gradients could shed light on a possible defense mechanism that these organisms could have adopted under physiological stress in their natural environment or in the laboratory setting to impart a protection against self-magnetics.
In this paper, we have found sudden motility reversals (Figure 1a) exhibited by the Magnetospirillum magneticum (AMB-1) that are related to magnetic field gradients. We proposed that these organisms may utilize these reversals to respond to magnetic disturbances.

\section{Materials and methods}

Helmholtz coils

In order to examine the interaction of AMB-1 cells, we custom built a pair of Helmholtz coils that produced an area with a uniform magnetic field (González et al., 2014) . This system consisted of two identical coils separated by the radius of the coils. The radius of the coil was 4.5 inch and the gauge of the magnet wire used was 22 AWG. For this pair of Helmholtz coils, there were 400 turns in each coil, and the windings were stacked in 7 layers. For the U-turn experiments, we used another larger pair of Helmholtz coils with a radius of 13 inch with 150 turns in each coil and the magnet wire gage used was 18 AWG. An Arduino Uno microcontroller was used to rapidly alternate the stimulation between a

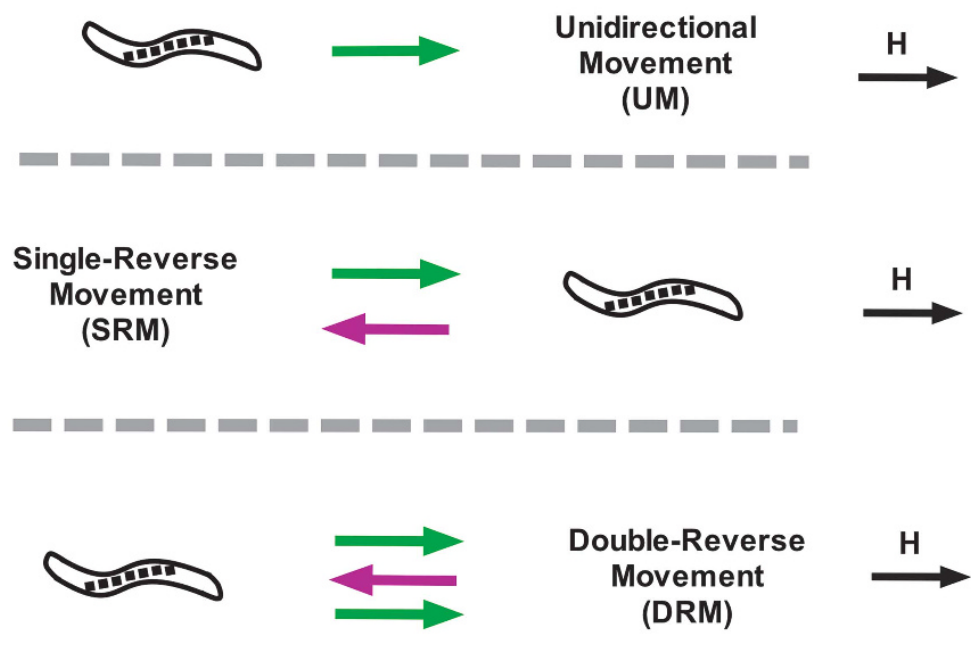

b
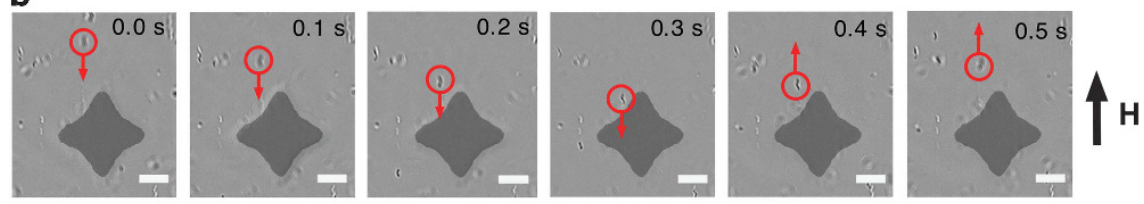

C
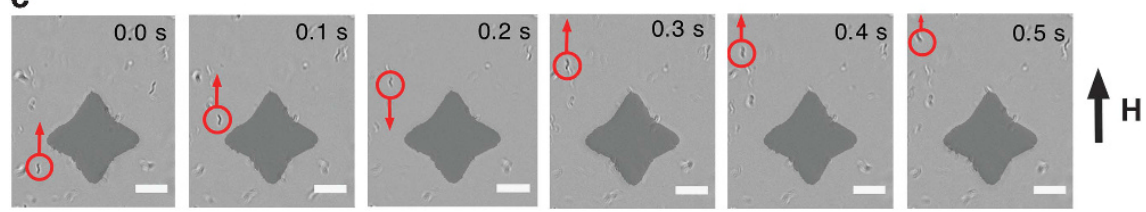

Figure 1 Observed reversals in magnetotactic bacteria in the vicinity of magnetic islands indicate magnetic field gradient sensing. (a) Schematic of the three observed modes of motility, UM, SRM and DRM, for AMB-1 approaching NiFe islands. Bright-field microscopy images of a time sequence of the motility path of representative AMB-1 approaching the magnetic islands and exhibiting (b) singlereverse and (c) double-reverse responses (scale bar, $5 \mu \mathrm{m}$ ). 
the two coils to do the U-turn experiments. A circuit with a transistor (TIP102) and diode (1N4004) was built to protect the electrical circuitry of the Arduino microcontroller as the maximum current the Arduino can take is $\sim 30 \mathrm{~mA}$ and we needed over $0.1 \AA$ to drive the coils. In the circuitry, we included light-emitting diodes (LEDs) to indicate which coils were operating at each time (Supplementary Figure S1 shows the coil arrangement and the circuit used to control both coils).

\section{Permalloy island fabrication}

Permalloy islands (Figures $1 \mathrm{~b}$ and c) were fabricated and then magnetized using the previously described Helmholtz coils. To visualize the interaction of the AMB-1 cells with these islands, we used a $\times 63$ objective on a modified inverted microscope (Zeiss Anxiovert 200, Göttingen, Germany). The permalloy islands and the microscope slide thickness was $<170 \mu \mathrm{m}$ for visualization. To make the permalloy island master mold, we used an image reversal fabrication process (Long and Newman, 1984). The ensuing master was used as the mold to deposit the metal. In order for the layer of permalloy to be optically transparent, the thickness of the metal needed to be $<30 \mathrm{~nm}$. A $25 \AA$ layer of tantalum and a $250 \AA$ thick layer of $\mathrm{NiFe}$ were subsequently deposited onto the master mold. The islands were rinsed in fresh acetone and isopropyl alcohol. Detailed information about the set-up and the fabrication of these islands can be found in the study of González et al. (2014).

\section{Analysis of the reversal behavior and direction of swimming}

To track the bacteria, we used a MATLAB code written by Wauthier (Wauthier, 2011). The code subtracts the background by determining the most frequent values of a pixel and it tracks the objects by gathering information from different frames using a Kalman filter. After running the run_tracker.m script, we used the extract_trajectories.m script to extract information such as the frame at which the cells were first and last observed, the position and the length of the trajectories and so on. This information was stored in structure (or struct) arrays. Using the MATLAB dot operator, these data were assessed. We used this information and wrote a MATLAB script to determine whether the cells were reversing and to determine the direction the cells were swimming (see script in the Supplementary Information).

\section{Results}

Reversal behaviors because of the magnetic islands Magnetotactic bacteria use their intracellular magnetic chains to align their movement to magnetic field lines, but the ability of these bacteria to switch directions in response to local magnetic field gradients has not been explored. Sudden motility reversals in axial magnetotactic bacteria have been previously observed in response to oxygen gradients in a mechanism known as magneto-aerotaxis, because it is a 'magnetically assisted aerotaxis' (Frankel et al., 1997). In this study, we imposed sharp concentrated magnetic field gradients using thin film permalloy $\left(\mathrm{Ni}_{80} \mathrm{Fe}_{20}\right)$ island $(\sim 30 \mathrm{~nm}$ in height) to concentrate magnetic fields generated through our custom-designed Helmholtz coils that produced a uniform magnetic field (González et al., 2014). We exposed AMB-1 cells to magnetic field gradients as they approached and crossed the starshaped islands. They experienced a change in the magnetic field from high to low and back to high again. The bacteria were observed to exhibit three distinct behavior modes, unidirectional movement (UM), single-reverse movement (SRM) and doublereverse movement (DRM), as shown in Figure 1a. The UM mode was characterized by continued, straight-line movement with only very slight deviations from the straight path in one direction as the AMB-1 cells moved toward and then across the patterned islands. This group of the AMB-1 cells responded with a UM mode and their movement was not affected by magnetic field gradients. However, another group of the bacteria exhibited a SRM mode, completely reversing their direction of motion as they reached the permalloy island and the associated magnetic field gradients (Figure 1b and Supplementary Movie S1). Other AMB-1 cells exhibited a DRM mode, in which the bacteria switched directions once in the vicinity of the islands, moving in the opposite direction for a short distance and then switching directions a second time to continue in their original direction (Figure $1 \mathrm{~b}$ and Supplementary Movie S2). A very small fraction of AMB-1 cells exhibited multiple reversals in sequence. These different reversals suggest these bacteria were detecting changes in the magnetic field or the magnetic field gradient.

Using the magnetic islands to create magnetic field gradients, we observed an unprecedented correlation between the magnetic field gradient and the reversing behavior in AMB-1 cells. The number of reversals (SRM and DRM) in the vicinity of the star-shaped island (Figure 2a) increased as we actuated the external field (from Helmholtz coils) from 0 to $\sim 13 \mathrm{mT}$. As an important control, we examined the motion of the bacteria when increasing the magnetic field, with no magnetic island present. The bacteria reversed only $\sim 6 \%$ of the time under this condition. At higher fields, $13 \mathrm{mT}, 42 \%$ of the AMB-1 cells exhibited the DRM or SRM motility mode, with $22 \%$ and $20 \%$ exhibiting the DRM and the SRM mode, respectively. The higher number of reversals at higher fields was noted to correspond to the location where the magnetic field varied significantly with position (that is, the region 
with a high magnetic field gradient) (Figures $2 \mathrm{~b}$ and c; see Supplementary Information for MATLAB script for analysis and quantification of the reversals).
We modeled the magnetic field using the finite element method package called FEMM (Finite Element Method Magnetics; see the Supplementary a

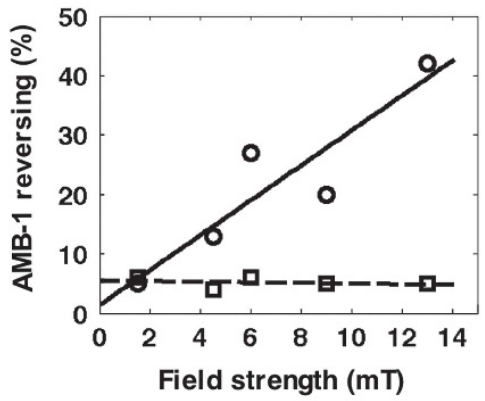

b

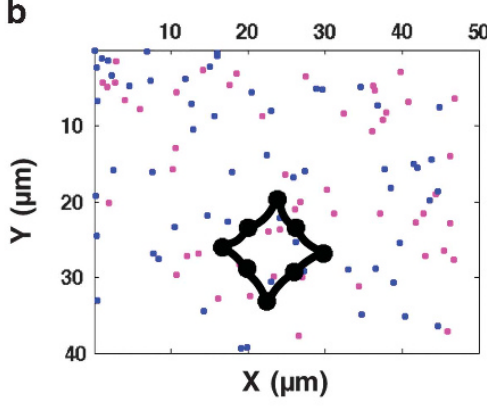

C
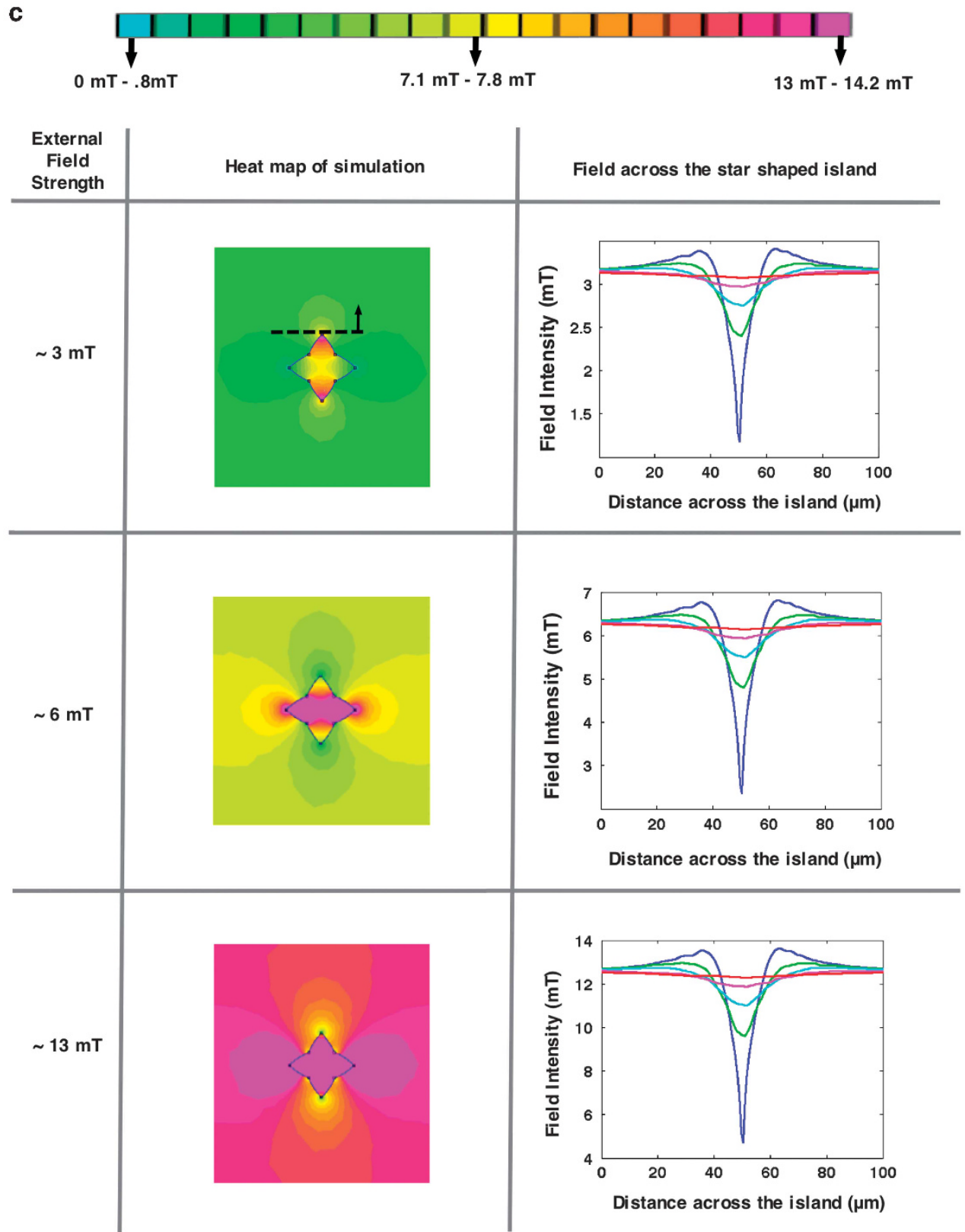
Information for details). At low fields, lower gradients were found $(\sim 3 \mathrm{mT}$ case; Figure $2 \mathrm{c})$, whereas at higher fields, higher gradients were present ( $\sim 13 \mathrm{mT}$ case; Figure 2c). We also examined the region in the vertical direction further away from the island's top tip (shown in Figure 2c with dashed lines in the $3 \mathrm{mT}$ case), which indicated the gradient decreased and the field became more uniform across the island (Figure 2c). It is interesting to note the degree of extension of the magnetic field lobes (showed the contour along which the magnetic field suddenly changes between the different regions) at different field magnitudes as shown in Figure 2c (simulation results). The higher the magnetic field was, the larger the area covered by these lobes. More interesting was the comparison of the $13 \mathrm{mT}$ case with Figure 2b (we plotted the first reversals for the $13 \mathrm{mT}$ case). In Figure 2b, there was an empty region where fewer SRM reversals were observed. This region resembled one of the lobes in the simulation where the magnetic field changed abruptly. We also determined the distribution of AMB-1 cells that exhibited different modes with respect to the speed of the bacteria. The reversing behavior was most often found among AMB-1 cells moving at $0-20 \mu \mathrm{m} \mathrm{s}^{-1}$ and less frequently for AMB-1 cells moving at higher speeds (see Supplementary Figure S2 in the Supplementary Information). Through simulation of the magnetic islands and comparison with experimental results, we reasoned that the motility reversals must be because of magnetic field gradients.

Swimming patterns of AMB-1 cells because of a permanent magnet

From the observation of the data collected with the magnetic islands and the Helmholtz coils, we speculated that the reversals were because of the magnetic field gradients. To further test our hypothesis, we reasoned that if the reversals are because of the magnetic field gradients then when using a permanent magnet with gradients throughout a larger surface area (that is, a global field), we should expect more reversals in comparison with that of the localized changes as it was in the case of the islands. To address this, we performed experiments using a permanent magnet and recorded whether the cells were swimming to the right or to the left assuming that they would be reversing near the edge as the magnet moved away from the cells (see Figure 3a for a schematic). We characterized the magnetic field gradient produced by the permanent magnet with respect to distance using a gaussmeter (Figure $3 \mathrm{~b}$ ) that was determined from the magnetic field versus distance plot shown in the inset of Figure $3 \mathrm{~b}$. We first determined the polarity of an iron permanent magnet (that is, which side of the magnet was north and south) using a compass and then recorded the percentage of the cells swimming away from the north (Figure 3c and Supplementary Movie S3; see Supplementary Table S1 for statistical analysis) and south faces (Figure 3d and Supplementary Movie S4; see Supplementary Table S2 for statistical analysis) of the magnet as we retracted the magnet from the droplet of cells. We imaged the cells at the center of the droplet and found that at 5 and $10 \mathrm{~mm}$, $\sim 70 \%$ of the cells were persistently swimming away from the magnet. This occurred when the north and south faces of the magnet were facing the droplet. At distances of $>30 \mathrm{~mm}$, the percentage of cells swimming away from the magnet decreased. This location corresponded to a gradient of $\sim 0.2 \mathrm{~T} \mathrm{~m}^{-1}$ (tesla per meter), whereas for the case of the magnetic island, we observed a higher magnetic field gradient of $\sim 7 \mathrm{~T} \mathrm{~m}^{-1}$. This suggests that closer to the island the cells experience larger gradients resulting in a direct visualization of the reversals. One point noted is that the $7 \mathrm{Tm}^{-1}$ gradients (at $\sim 15 \mathrm{~mm}$ from the island) could be beyond the threshold and a $0.2 \mathrm{Tm}^{-1}$ gradient is close to the threshold that induces this behavior.

\section{Alignment of the bacteria to the Helmholtz coils}

In the absence of an external magnetic field, the AMB-1 cells showed no preferred direction of movement in the field of view (Figures $4 a$ and $b$ and Supplementary Movie S5). Upon application of a uniform magnetic field $(\sim 1 \mathrm{mT})$, the bacteria aligned to the direction of the magnetic field. This alignment alteration was likely because of a forceinduced torque that caused the bacteria to rotate (Figures 4a and c and Supplementary Movie S6). In addition, when we increased the magnetic field from 0 to $1.6 \mathrm{mT}$, the fraction of bacteria aligning their movement to the direction of the magnetic

Figure 2 Augmentation of the reversals with increasing external Helmholtz coils' magnetic field and simulation of a star-patterned island at different magnetic field strengths. (a) The number of reversals (SRM and DRM) in the vicinity of a star-patterned island increases linearly with field strength. This is plotted as a percentage and it is represented by the circle symbols. The control is the percentage of the reversals in the presence of an external magnetic field without islands (square symbols). The lines are the linear fit to the points. (b) The position of the reversals with respect to the star-patterned island. The island is represented by the black dots. The blue and magenta dots represent the location of the bacteria when they reverse and swim to the left (blue dots) and to the right (magenta dots) for $\sim 13 \mathrm{mT}$. (c) Simulation of the magnetic field response for the star island upon exposure of different magnetic fields (3, 6 and $13 \mathrm{mT}$ ) from Helmholtz coils. This figure shows a magnetic field heat map for each field case to show the extension of the magnetic field lobes (the regions where the magnetic field changes). As the field increases the area of the lobes increases. This figure also shows the field intensity (in mT) across the top of the island at $0 \mu \mathrm{m}$ (blue), $5 \mu \mathrm{m}$ (green), $10 \mu \mathrm{m}$ (cyan), $20 \mu \mathrm{m}$ (magenta) and $43 \mu \mathrm{m}$ (red). The top of the tip of island is indicated by dashed line shown only in the $3 \mathrm{mT}$ case. As the external magnetic field increases, there is higher drop in the field across the island, indicating the increase in the field gradient. Far away from the island's top tip, the gradient decreases and the field becomes more uniform across the island. 


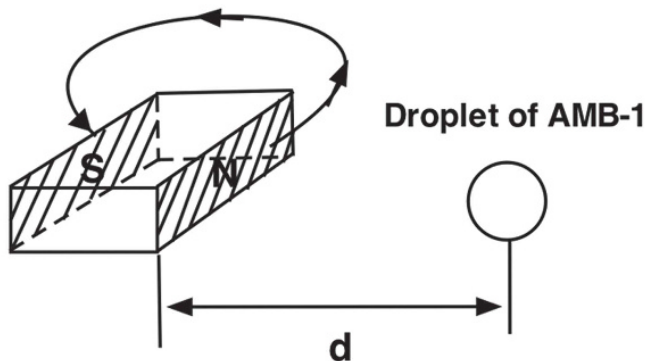

C

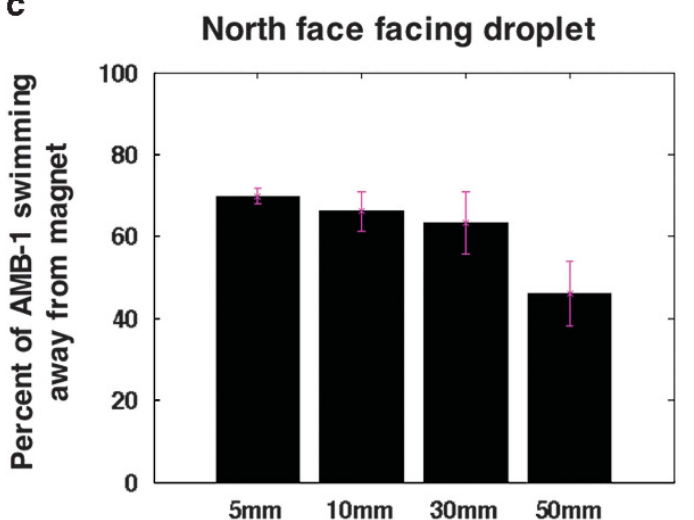

b

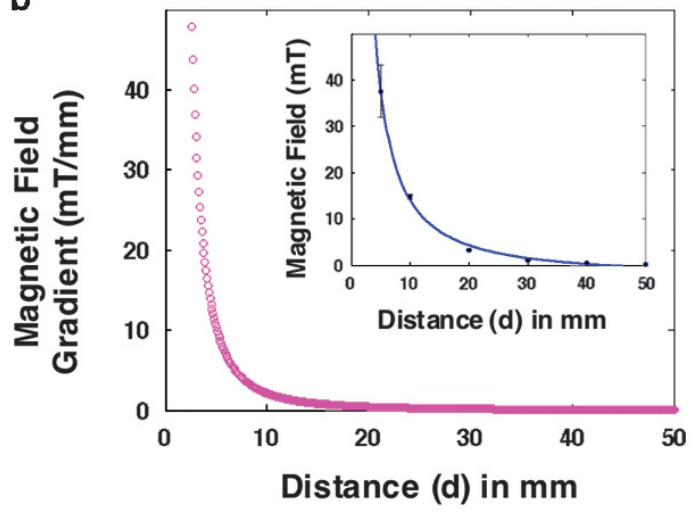

d

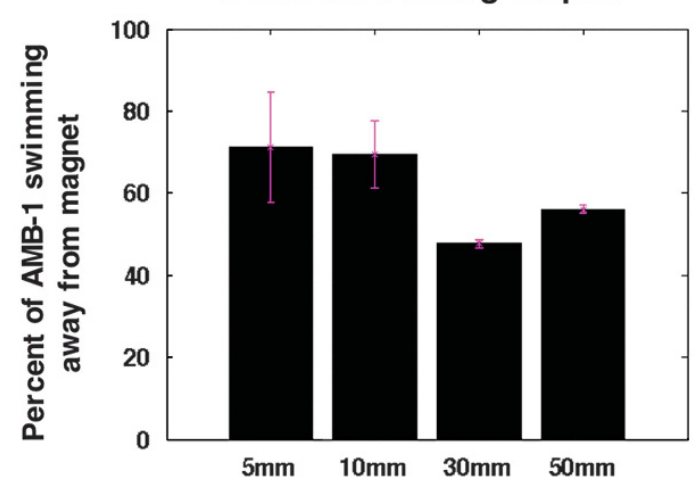

Figure 3 Swimming effect using a permanent magnet in close proximity to a droplet of AMB-1s. (a) Schematic of the position of the permanent magnet relative to a droplet of cells. The distance, $d$, is the gap between the edge of the magnet and the center of the droplet of cells. (b) Characterization of the magnetic field gradient of the iron permanent magnet with respect to the distance (in magenta). The inset shows the magnetic fields corresponding to each point in the magnetic gradient field vs distance plot. The percentage of AMB-1 swimming away from the magnet for the corresponding distance of $5,10,30$ and $50 \mathrm{~mm}$ when the permanent magnet's north face (c) is facing the droplet and when the south face (d) is facing the droplet. There is a threshold at which the bacteria are no longer persistently swimming away from the magnet. This distance is at distance $>30 \mathrm{~mm}$. This distance corresponds to approximately where the gradient approaches zero. Table S1 in the Supplementary Information presents the $P$-values corresponding to each data set here showing the statistical significance for the groups.

field increased from 0 to $\sim 90 \%$ (Figure $4 \mathrm{~d}$ ). Above $1.1 \mathrm{mT}$, the fraction of bacteria aligning to the magnetic field did not increase further. In this case, the threshold at $\sim 1.1 \mathrm{mT}$ may induce a force sufficiently strong so that all of the bacteria can no longer overcome an applied torque on the magnetosome chain (Supplementary Figure S3 shows the transmission electron microscopy images and analysis). The variation in the number of particles within each cell could account for the difference in alignment of the cells to the external magnetic field.

\section{U-turn versus reversals}

Comparing the reversal behaviors with that of the U-turn was important to clearly differentiate between the two movements. Previous work has documented U-turn motion (Esquivel and Lins De Barros, 1986) observed when subjecting magnetotactic bacteria to a reversing magnetic field (Frankel, 2003). Our reported reversing behavior in the presence of the induced local magnetic field gradients is very different from these U-turn responses. To examine this further, we built on previous work that analyzed time to reverse, $\tau$, and U-turn width, $W$, for spirillum organisms (see Supplementary Information). Similar expressions have been developed for a coccus organism (Esquivel and Lins De Barros, 1986) and nonspherical magnetotactic bacteria (Bahaj et al., 1996). These papers predicted that for an AMB-1 cell to make a U-turn that is $\sim 10 \mu \mathrm{m}$ in width with an applied field of $\sim 1 \mathrm{mT}$, the time to make the U-turn is $\tau \sim 0.5 \mathrm{~s}$. We first confirmed these predictions through experiments in which we completely reversed the field and measured the time it took for the bacteria to make a U-turn (Supplementary Figure S4 and Supplementary Movie S7). In contrast, our findings (Figure 1) showed that in the vicinity of high magnetic field gradients, the AMB-1 cells reversed without turning around, suggesting a different mechanism. To reverse direction, the bacteria can change the propulsion from the flagellum on one end of its body to the flagellum on the opposite end (Krieg, 1976; Thar and Fenchel, 2005). 


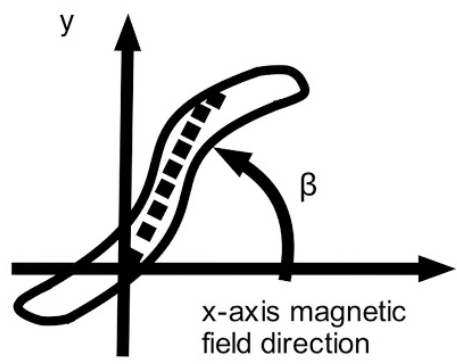

c

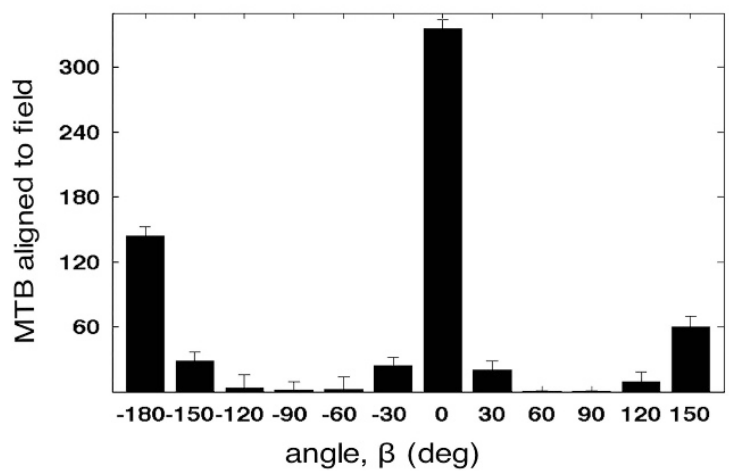

b

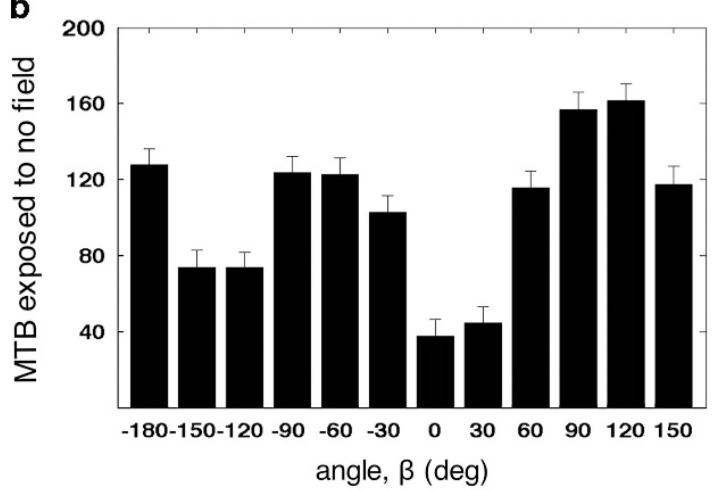

d

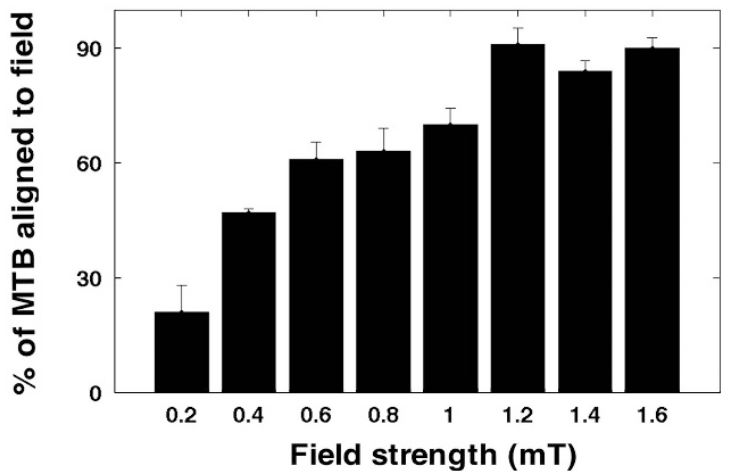

Figure 4 Alignment of AMB-1 during global external magnetic field application. (a) Schematic of coordinates indicating the angle (in b, c) of the AMB-1 cell body with respect to the positive $\mathrm{x}$ axis, the direction of the magnetic field direction. (b) Histogram of the orientation of AMB-1 motility direction with no external applied magnetic field (see (a) for angle). (c) Histogram of the orientation of AMB-1 motility with $1.0 \mathrm{mT}$ external magnetic field applied along the $\mathrm{x}$ axis (see (a) for angle). (d) Percentage of MTB aligned at $0^{\circ}$ with increasing externally applied magnetic field strength. A maximum response (saturation) appears to occur at $\sim 1.1 \mathrm{mT}$ where $90 \%$ of the bacteria are completely aligned with the direction of the external magnetic field.

\section{Discussion}

Alignment to an external magnetic field

The uniformity of magnetic fields is important when studying magnetotactic bacteria. It is worthwhile to reiterate that Helmholtz coils provide a uniform magnetic field and the introduction of a magnetic material in the pool of these magnetic field lines causes them to warp and penetrate or permeate the magnetic materials such as the permalloy islands. However, a permanent magnet found in nature always produces a magnetic field gradient. Others have observed similar reversal behaviors with different magnetotactic organisms under different conditions. For example, Greenberg reported 'ping-pong' movements associated with magnetotactic multicellular organisms (Greenberg et al., 2005). Erglis et al. (2007) also observed a 'back and forth' motion when studying the dynamics of Magnetospirillum gryphiswaldense in a rotating uniform magnetic field. An early study by Spormann and Wolfe (1984) called a observed reversal behavior 'tactile behavior,' and they demonstrated the maintenance of a band of cells while aligning the cells with a magnet. These results have similar components to our findings with the permanent magnet as at a certain distance, the cells start swimming to the left and to the right at about equal number. Frankel et al. (1997) showed that axial magnetotactic bacteria use the magnetic field lines as an axis and occasionally switch direction as they swim, and they concluded that these bacteria use a temporal sensory mechanism sampling the media and swimming up an oxygen gradient that they called two-way swimming. All of the studies mentioned above used various types of fields from rotating fields to uniform to permanent magnets. The type and nature of the magnetic field applied while doing experiments with MTB is crucial to correctly determine the effect of other environmental cues as these cells sense magnetic field gradients as demonstrated in this study.

\section{Evolutionary implication and the ecology}

In the literature, it is hypothesized that magnetosomes impart MTB with an advantage in finding favorable environments in an efficient matter by using a simple route for obtaining nutrients rather than the more widely used, well-known and random walks employed by some other organisms such as 
Escherichia coli (Berg, 1993). MTBs in the Northern Hemisphere and Southern Hemisphere predominantly swim toward the north and south, respectively (Blakemore, 1975; Blakemore et al., 1980), that helps the cells to swim in a roughly vertical pathway downward toward the oxygen-depleted region near the sediments. However, these observations did not explain the benefit of alignment and the predominance of magnetotactic bacteria in the oxic-anoxic transition zone, which forms from an inverse double gradient of oxygen $\left(\mathrm{O}_{2}\right)$ from above and sulfide $\left(\mathrm{S}^{2-}\right)$ from below. This zone is scant in oxygen and sulfide concentrations (Simmons et al., 2004). Moreover, south-seeking cells were once found in the Northern Hemisphere (Simmons et al., 2006), conflicting with previous studies. Our study attempts to explain these discrepancies and indicates that determining the uniformity of the magnetic field is extremely important before drawing conclusions as MTB may respond to magnetic field gradients. The magnetoaerotaxis theory emerged to explain the predominance of MTB in the oxic-anoxic transition zone. The theory suggests that these organisms swim to find comfortable oxygen gradient using the Earth's magnetic field as an axis as in a 'magnetically assisted aerotaxis' (Frankel et al., 1997). The magneto-aerotaxis theory provides a more reasonable explanation, but the system could be more complex than this as these organisms may be sensing magnetic field gradients.

Despite all of the studies involving MTB organisms, the sensing mechanism and the connection of the magnetosome structures to the flagella motor are largely unknown. One possible aspect of this unknown magnetosome-flagella system is a possible mechanism developed by these organisms to cope with self-magnetics against magnetic fluctuations in their environments. For example, if a MTB is in the vicinity of a large volume of magnetite, it could be immobilized because of its magnetic body. There is a report on the dynamics of the MTB populations found in chemically stratified water column presumably because of water column chemistry that moves the oxic-anoxic transition zone throughout the seasons, and at some point it gets much closer to the sediments (Simmons et al., 2007). Taking this into account along with the abundance of MTB and the accumulation and preservation of these in the sediments, termed magnetofossils, Kopp and Kirschvink (2008) could indicate an important role in the selection of bacteria with the ability to keep a distance from magnetic sediments that could immobilize them. The disintegration of the organic constituents is likely occurring and the sediments could contain clumps and agglomeration of magnetic specimens (Simmons et al., 2007; Kopp and Kirschvink, 2008). This may present a threat to these organisms. In addition, studies have shown the preservation of magnetofossils with only minimal extensive dissolution effect of Deep Sea Drilling Projects
(Vali and Kirschvink, 1989), which aids in the conservation of the magnetic properties.

Furthermore, during the Paleocene-Eocene Thermal Maximum time interval, with abundant bacterial magnetofossils, Schumann et al. (2008) found a giant spearhead-like shape up to $4 \mu \mathrm{m}$ long and that most likely derived from eukaryotes (because of their large size). Magnetofossils usually appear in aggregates of several dozen particles (Petersen et al., 1986; Stolz et al., 1986), increasing the volume of the magnetic material and thus the magnetic field emanating from these species. These kinds of magnetofossils could contribute to the development of MTB mechanism to cope with self-magnetics and to dodge magnetic attractive forces by swimming away from magnetic debris. For these cells, having a magnetic body could be a burden under the presence of any magnetic material. The AMB-1 cells could be displaying this intrinsic property through the abrupt changes in direction previously observed.

\section{MCP and the flagella motor}

In addition, the reversal behaviors could involved a more complex magnetoreception mechanism (Kirschvink et al., 2001). For example, this microorganism has a significant number of expressed methyl-accepting chemotaxis proteins (MCPs) (Fukuda et al., 2006) that are not thoroughly understood in terms of their interactions with the magnetosome chain. Philippe and Wu (2010) suggested a chemotaxis-like magnetotaxis model that may be related to the switching behavior observed in this study. MCP Amb0994 is one of the two MCPs found within the magnetosome island (MAI) that harbors proteins known to be involved in magnetosome formation and biomineralization (Murat et al., 2010). The theory is that the magnetosome chain could be affected by MCP Amb0994, but the mechanism has yet to be elucidated. In this model, when a magnetic torque is imposed on the MamK, which is a cytoskeleton protein involved in giving the magnetosome chain its structural scaffold (Komeili et al., 2006; Pradel et al., 2006; Scheffel et al., 2006), it presumably interacts with a MCP, Amb0994 (which lacks a periplasmic sensing domain; presumably receiving an internal signal) (Philippe and $\mathrm{Wu}, 2010$ ). This MCP protein in turn could be relaying a signal to the flagella motor switch protein, FliM, through an unknown mechanism or through a CheA-CheY signal transduction system (Falke and Hazelbauer, 2001; West and Stock, 2001; Victor, 2004, Wadhams and Armitage, 2004), presumably slowing down the bacteria. The bacteria showing SRM and DRM are slower than the ones showing UM, and protein Amb0994 and other associate genes (Greene et al., 2012) could be responsible for these reversals as this protein localizes at the poles near the flagella motor. Amb0994 could be implicated in the sudden reversal behavior responses that we have observed 
in AMB-1 cells in the presence of magnetic field gradients.

Temporal versus spatial sensing in AMB-1 cells Cells detect gradients through two types of mechanisms: temporal and spatial. It is widely known that small bacteria usually employ a temporal sensing mechanism to sense changes in the environment by sampling the concentration and integrating the signal over time because of the limitation in their size (Macnab and Koshland, 1972). However, bacteria can also employ a spatial sensing mechanism (Dusenbery, 1998; Thar and Kühl, 2003; Hu et al., 2010; Lower et al., 2010). In a spatial detection mechanism, a cell compares the difference between different receptors and instantly makes a decision based on these various inputs. We propose the AMB-1 cells perform reversals by employing a spatial detection mechanism to instantly sense magnetic field gradients. This could be best explained through changes in the curvature of the field lines as shown in Figure 5. Hu et al. (2010) argue that receptor coupling can improve sensing of a gradient in bacteria by spatial sensing. In addition, Dusenbery (1998) argues that when steep gradients occur, spatial sensing is favorable over temporal sensing. The changing magnetic fields can exert a bending force on the magnetosome chains (Figure 5)
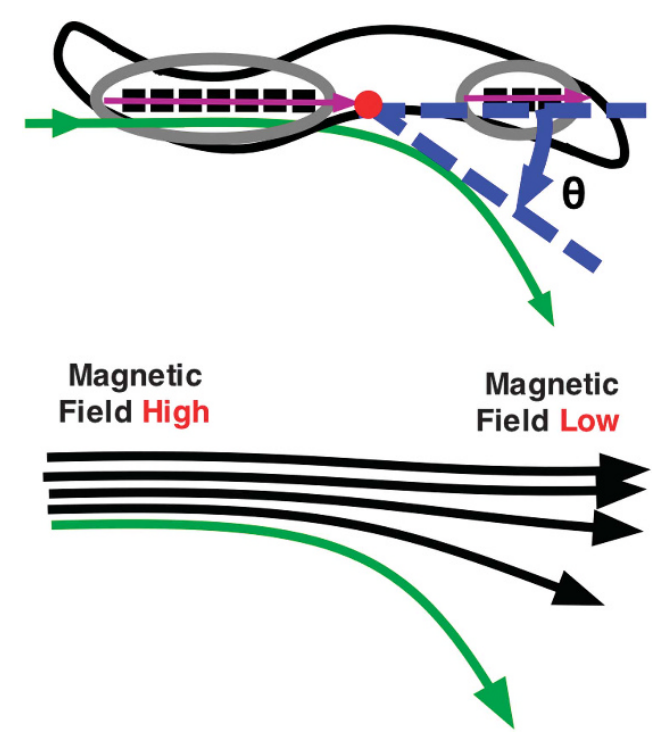

Figure 5 Schematic of the potential physical force application on the magnetosome chain of the magnetotactic bacteria due to a magnetic field gradient. The force could be bending a bacterium slightly through a change in the force when the field lines diverge as the magnetic field decreases. The tail of a bacterium could remain aligned to the field while the head swivels or rotates (through angle $\theta$ in the diagram). The red dot in this schematic would indicate the center of mass for the rotation to attempt to align to the magnetic field lines that are spreading apart (green line). This spreading of the magnetic field lines from being in parallel and closer together is because of the magnetic field lines tendency to penetrate a magnetic object such as the permalloy island. that can be in turn transferred through a coupling protein. The MamK could be acting as a coupling protein as it forms long filaments along the length of the cells body between the two cell poles of the AMB-1 cells. MamK could be interacting with MCP Amb0994 at the poles to allow a fast relay of the magnetic signal between the head and the tail and establish a communication between the two flagella motors. The Helmholtz coils produce straight lines and as these approach the islands they tend to bend toward the island (as shown in the simulation of Figure 2). These bending of the field lines could be causing the cells to sense different curvature of the field lines at the head and at the tail of their long bodies. This difference in the field could be torqueing the cells and relaying this signal to the MCP Amb0994. This could be related to the sensing of magnetic field line angles proposed by Zhu et al. (2014) as they proposed a similar model.

\section{Conclusions}

In this study, we found that AMB-1 cells posses the ability to sense magnetic field gradients and respond to them by reversing direction. We investigated this sensing capability by exposing the AMB-1 cells to magnetic field gradients from magnetic islands and Helmholtz coils. Motile AMB-1 cells swim away from the north and south poles of a permanent magnet, which also indicate sensing of magnetic field gradients. Our results indicate that the magnetic field sensing mechanisms of these organisms may be even more sophisticated than previously realized, and the system provides these AMB-1 cells a pivotal capability that we call protection against self-magnetics.

\section{Conflict of Interest}

The authors declare no conflict of interest.

\section{Acknowledgements}

We acknowledge Joseph Suhan for his help in acquiring TEM images. We also thank Sara Majetich for her valuable discussion about the manuscript. This work was supported in part by the Office of Naval Research (N000140910215), the Sloan Foundation, the Air Force Office of Scientific Research (FA9550-13-1-01-08) as well as a National Science Foundation Graduate Research Fellowship under Grant No. 0946825 to LMG. In addition, we want to acknowledge the National Science Foundation with Grant No. CMMI1100430, CMMI-1160840 and CPS-1135850.

\section{References}

Bahaj AS, James PAB, Moeschler FD. (1996). An alternative method for the estimation of the magnetic moment of non-spherical magnetotactic bacteria. IEEE Trans Magn 32: 5133-5135. 
Berg H. (1993). Random Walks in Biology. Princeton University Press.

Blakemore R. (1975). Magnetotactic bacteria. Science 190: $377-379$.

Blakemore RP, Frankel RB, Kalmijn AJ. (1980). South-seeking magnetotactic bacteria in the Southern Hemisphere. Nature 286: 384-385.

Cullity BD, Graham CD. (2009). Introduction to Magnetic Materials. Wiley-IEEE Press: Hoboken.

Diebel CE, Proksch R, Green CR, Neilson P, Walker MM. (2000). Magnetite defines a vertebrate magnetoreceptor. Nature 406: 299-302.

Dusenbery DB. (1998). Spatial sensing of stimulus gradients can be superior to temporal sensing for free-swimming bacteria. Biophys J 74: 2272-2277.

Erglis K, Wen Q, Ose V, Zeltins A, Sharipo A, Janmey PA et al. (2007). Dynamics of magnetotactic bacteria in a rotating magnetic field. Biophys J 93: 1402-1412.

Esquivel DMS, Lins De Barros HGP. (1986). Motion of magnetotactic microorganisms. J Exp Biol 121: 153-163.

Falke JJ, Hazelbauer GL. (2001). Transmembrane signaling in bacterial chemoreceptors. Trends Biochem Sci 26: 257-265.

Frankel RB, Blakemore RP, Wolfe RS. (1979). Magnetite in freshwater magnetotactic bacteria. Science 203: 1355-1356.

Frankel RB, Bazylinski DA, Johnson MS, Taylor BL. (1997). Magneto-aerotaxis in marine coccoid bacteria. Biophys J 73: 994-1000.

Frankel RB. (2003). Magnetic guidance of organisms. Annu Rev Biophys Bioeng 13: 85-103.

Frankel RB, Williams T. J, Bazylinski D. A. (2006). Magneto-aerotaxis. In: Schuler D (ed) Magnetoreception and Magnetosome in Bacteria. Springer: New York, pp 1-24.

Fukuda Y, Okamura Y, Takeyama H, Matsunaga T. (2006). Dynamic analysis of a genomic island in Magnetospirillum sp. strain AMB-1 reveals how magnetosome synthesis developed. FEBS Lett 580: 801-812.

González LM, Ruder WC, LeDuc PR, Messner WC. (2014). Controlling magnetotactic bacteria through an integrated nanofabricated metallic island and optical microscope approach. Sci Rep 4: 1-8.

Gould JL, Kirschvink JL, Deffeyes KS. (1978). Bees have magnetic remanence. Science 201: 1026-1028.

Greenberg M, Canter K, Mahler I, Tornheim A. (2005). Observation of magnetoreceptive behavior in a multicellular magnetotactic prokaryote in higher than geomagnetic fields. Biophys J 88: 1496-1499.

Greene SE, Brilli M, Biondi EG, Komeili A. (2012). Analysis of the CtrA pathway in magnetospirillum reveals an ancestral role in motility in alphaproteobacteria. J Bacteriol 194: 2973-2986.

Hu B, Chen W, Rappel W-J, Levine H. (2010). Physical limits on cellular sensing of spatial gradients. Phys Rev Lett 105: 048104.

Katzmann E, Scheffel A, Gruska M, Plitzko JM, Schüler D. (2010). Loss of the actin-like protein MamK has pleiotropic effects on magnetosome formation and chain assembly in Magnetospirillum gryphiswaldense. Mol Microbiol 77: 208-224.

Kirschvink JL, Walker MM, Diebel CE. (2001). Magnetite-based magnetoreception. Curr Opin Neurobiol 11: $462-467$.

Komeili A, Vali H, Beveridge TJ, Newman DK. (2004). Magnetosome vesicles are present before magnetite formation, and MamA is required for their activation. Proc Natl Acad Sci USA 101: 3839-3844.

Komeili A, Li Z, Newman DK, Jensen GJ. (2006). Magnetosomes are cell membrane invaginations organized by the actin-like protein MamK. Science 311: 242-245.

Komeili A. (2012). Molecular mechanisms of compartmentalization and biomineralization in magnetotactic bacteria. FEMS Microbiol Rev 36: 232-255.

Kopp RE, Kirschvink JL. (2008). The identification and biogeochemical interpretation of fossil magnetotactic bacteria. Earth Sci Rev 86: 42-61.

Krieg NR. (1976). Biology of the chemoheterotrophic spirilla. Bacteriol Rev 40: 55-115.

Long ML, Newman J. (1984). Image reversal techniques with standard positive photoresist. PrOC SPIE 0469: 189-193.

Lowenstam HA. (1981). Minerals formed by organisms. Science 211: 1126-1131.

Lower SK, Yongsunthon R, Casillas-Ituarte NN, Taylor ES, DiBartola AC, Lower BH et al. (2010). A tactile response in Staphylococcus aureus. Biophys $J$ 99: 2803-2811.

Macnab RM, Koshland DE. (1972). The gradient-sensing mechanism in bacterial chemotaxis. Proc Natl Acad Sci USA 69: 2509-2512.

Maher BA. (1998). Magnetite biomineralization in termites. Proc $R$ Soc London $B$ Biol Sci 265: 733-737.

Mann S, Sparks NH, Walker MM, Kirschvink JL. (1988). Ultrastructure, morphology and organization of biogenic magnetite from sockeye salmon, Oncorhynchus nerka: implications for magnetoreception. J Exp Biol 140: $35-49$.

Murat D, Quinlan A, Vali H, Komeili A. (2010). Comprehensive genetic dissection of the magnetosome gene island reveals the step-wise assembly of a prokaryotic organelle. Proc Natl Acad Sci USA 107: 5593-5598.

Petersen N, von Dobeneck T, Vali H. (1986). Fossil bacterial magnetite in deep-sea sediments from the South Atlantic Ocean. Nature 320: 611-615.

Philippe N, Wu L. (2010). An MCP-like protein interacts with the MamK cytoskeleton and is involved in magnetotaxis in Magnetospirillum magneticum AMB-1. J Mol Biol 400: 309-322.

Pradel N, Santini C, Bernadac A, Fukumori Y, Wu L. (2006). Biogenesis of actin-like bacterial cytoskeletal filaments destined for positioning prokaryotic magnetic organelles. Proc Natl Acad Sci USA 103: 17485-17489.

Ruder WC, Hsu C-PD, Edelman BD, Schwartz R, LeDuc PR. (2012). Biological colloid engineering: Self-assembly of dipolar ferromagnetic chains in a functionalized biogenic ferrofluid. Appl Phys Lett 101: 063701.

Scheffel A, Gruska M, Faivre D, Linaroudis A, Plitzko JM, Schuler D. (2006). An acidic protein aligns magnetosomes along a filamentous structure in magnetotactic bacteria. Nature 440: 110-114.

Schumann D, Raub TD, Kopp RE, Guerquin-Kern J-L, $\mathrm{Wu}$ T-D, Rouiller I et al. (2008). Gigantism in unique biogenic magnetite at the Paleocene-Eocene Thermal Maximum. Proc Natl Acad Sci USA 105: 17648-17653.

Simmons SL, Sievert SM, Frankel RB, Bazylinski DA, Edwards KJ. (2004). Spatiotemporal distribution of marine magnetotactic bacteria in a seasonally 
stratified coastal salt pond. Appl Environ Microbiol 70: 6230-6239.

Simmons SL, Bazylinski DA, Edwards KJ. (2006). South-seeking magnetotactic bacteria in the Northern Hemisphere. Science 311: 371-374.

Simmons SL, Bazylinski DA, Edwards KJ. (2007). Population dynamics of marine magnetotactic bacteria in a meromictic salt pond described with qPCR. Environ Microbiol 9: 2162-2174.

Spormann AM, Wolfe RS. (1984). Chemotactic, magnetotactic and tactile behaviour in a magnetic spirillum. FEMS Microbiol Lett 22: 171-177.

Stolz JF, Chang S-BR, Kirschvink JL. (1986). Magnetotactic bacteria and single-domain magnetite in hemipelagic sediments. Nature 321: 849-851.

Thar R, Kühl M. (2003). Bacteria are not too small for spatial sensing of chemical gradients: an experimental evidence. Proc Natl Acad Sci USA 100: 5748-5753.

Thar R, Fenchel T. (2005). Survey of motile microaerophilic bacterial morphotypes in the oxygen gradient above a marine sulfidic sediment. Appl Environ Microbiol 71: 3682-3691.
Vali H, Kirschvink JL. (1989). Magnetofossil dissolution in a palaeomagnetically unstable deep-sea sediment. Nature 339: 203-206.

Victor S. (2004). Receptor clustering and signal processing in E. coli chemotaxis. Trends Microbiol 12: 569-576.

Wadhams GH, Armitage JP. (2004). Making sense of it all: bacterial chemotaxis. Nat Rev Mol Cell Biol 5: 1024-1037.

Walcott C. (1977). Magnetic fields and the orientation of homing pigeons under sun. J Exp Biol 70: 105-123.

Walcott C, Gould JL, Kirschvink JL. (1979). Pigeons have magnets. Science 205: 1027-1029.

Wauthier F. (2011). MATLAB code for motion tracking in video sequences [Computer Program]. Available at: http://www.stats.ox.ac.uk/ wauthier/tracker/trackercode-2011.11.18.zip.

West AH, Stock AM. (2001). Histidine kinases and response regulator proteins in two-component signaling systems. Trends Biochem Sci 26: 369-376.

Zhu X, Ge X, Li N, Wu L-F, Luo C, Ouyang Q et al. (2014). Angle sensing in magnetotaxis of Magnetospirillum magneticum AMB-1. Integr Biol 6: 706-713.

Supplementary Information accompanies this paper on The ISME Journal website (http://www.nature.com/ismej) 